\title{
HALÁSZ'S THEOREM FOR BEURLING GENERALIZED NUMBERS
}

\author{
GREGORY DEBRUYNE, FREDERICK MAES, AND JASSON VINDAS
}

\begin{abstract}
We show that Halász's theorem holds for Beurling numbers under the following two mild hypotheses on the generalized number system: existence of a positive density for the generalized integers and a Chebyshev upper bound for the generalized primes.
\end{abstract}

\section{INTRODUCTION}

Halász's theorem [9] is a cornerstone in classical probabilistic number theory [8, 15]. This important result has been generalized by several authors [11, 16] to the context of abstract analytic number theory; the most general version so far being the one recently obtained by Zhang for Beurling numbers in [17].

Let $1<p_{1} \leq p_{2} \leq \ldots$ be a Beurling generalized prime number system. Its associated set of generalized integers (cf. [1, 2, 1, 12]) is the multiplicative semigroup generated by 1 and the generalized primes, which we arrange in a non-decreasing sequence taking multiplicities into account, $n_{0}=1<n_{1} \leq n_{2} \leq n_{3} \leq \ldots$ Denote as $N$ and $\pi$ the counting functions of the generalized integers and primes. As in classical number theory, we consider the weighted prime counting functions

$$
\Pi(x)=\sum_{p_{k}^{\alpha_{k} \leq x}} 1 / \alpha_{k} \quad \text { and } \quad \psi(x)=\int_{1}^{x} \log u \mathrm{~d} \Pi(u) .
$$

Given a function of (local) bounded variation $G$, we denote its Mellin-Stieltjes transform as $\widehat{G}(s)=\int_{1^{-}}^{\infty} x^{-s} \mathrm{~d} G(x)$ and we use the notation $s=\sigma+i t$ for complex variables.

Zhang's version of Halász's theorem reads as follows. His result generalizes [5, Theorem 3.1], where the set of hypotheses (1.1), (1.2), and (1.3) were actually introduced.

Theorem 1.1 (Zhang [17]). Suppose that the generalized number system satisfies a Chebyshev upper estimate

$$
\psi(x) \ll x,
$$

2010 Mathematics Subject Classification. Primary 11N37, 11N80; Secondary 11N05, 11N64, 11M41.

Key words and phrases. Halász theorem; Halász-Wirsing mean-value theorem; Beurling generalized primes and integers; multiplicative functions; multiplicative arithmetic measures; mean-value vanishing of the Möbius function.

G. Debruyne gratefully acknowledges support by a Postdoctoral Fellowship of the Research Foundation-Flanders.

The work of J. Vindas was supported by the Research Foundation-Flanders, through the FWOgrant number $1510119 \mathrm{~N}$. 
the generalized numbers have positive density

$$
N(x) \sim a x
$$

for some $a>0$, and $\left(\sigma \rightarrow 1^{+}\right)$

$$
\int_{1}^{\infty} \frac{|N(x)-a x|}{x^{\sigma+1}} \mathrm{~d} x \ll(\sigma-1)^{-\beta} \quad \text { for some } \beta \in[0,1 / 2) \text {. }
$$

Let $g$ be a completely multiplicative function such that $\left|g\left(n_{k}\right)\right| \leq 1$ for each $n_{k}$ and set $G(x)=\sum_{n_{k} \leq x} g\left(n_{k}\right)$. Then,

$$
G(x) \sim c x
$$

if and only if

$$
\widehat{G}(s)=\frac{c}{s-1}+o\left(\frac{1}{\sigma-1}\right)
$$

uniformly for $t$ on compact intervals.

The aim of this article is to considerably improve Theorem 1.1. We shall show that it still holds if one removes the condition (1.3) from its hypotheses. In addition to hold under weaker assumptions, our results are somewhat more general as they also involve slowly varying functions in the asymptotic formulas and apply to multiplicative functions on non necessarily discrete number systems. We mention that our method here is inspired by the treatment of Schwarz and Spilker from [14] of the DaboussiIndlekofer elementary proof [4] of the classical Halász theorem.

Finally, it should be pointed out that our considerations yield the following improvement to [5, Theorem 3.1], where $M$ is the sum function of the Möbius function of a generalized number system.

Corollary 1.2. The positive density condition (1.2) and the Chebyshev upper bound (1.1) imply the estimate $M(x)=o(x)$.

\section{Main Result And SOME CONSEQUENCES}

Let us start with our definition of the analog of a multiplicative function on a non necessarily discrete generalized number system. In a broader sense [2, 7], a Beurling generalized number system is merely a pair of non-decreasing right continuous functions $N$ and $\Pi$ with $N(1)=1$ and $\Pi(1)=0$, both having support in $[1, \infty)$, and subject to the relation $\mathrm{d} N=\exp ^{*}(\mathrm{~d} \Pi)$, where the exponential is taken with respect to the (multiplicative) convolution of measures [7]. Since the hypotheses used in this article always guarantee convergence of the Mellin transforms, the latter becomes equivalent to the zeta function identity

$$
\zeta(s)=\int_{1^{-}}^{\infty} x^{-s} \mathrm{~d} N(x)=\exp \left(\int_{1}^{\infty} x^{-s} \mathrm{~d} \Pi(x)\right) .
$$

We shall say that a (complex-valued) measure $\mathrm{d} G$ (supported on $[1, \infty))$ is arithmetic (w.r.t. the number system under consideration) if it is absolutely continuous with respect to $\mathrm{d} N$. Furthermore, we call it multiplicative if it can be written as $\mathrm{d} G=$ $\exp ^{*}(g \mathrm{~d} \Pi)$ for some function $g$. Clearly, every multiplicative measure is arithmetic. 
We can now state the main result of this article, its proof will be postponed to Section 4 .

Theorem 2.1. Suppose the number system satisfies the upper and lower logarithmic density conditions

$$
\int_{1^{-}}^{x} \frac{\mathrm{d} N(u)}{u} \asymp \log x .
$$

Let $\mathrm{d} G=\exp ^{*}(g \mathrm{~d} \Pi)$ be a multiplicative arithmetic measure with $g=g_{1}+g_{2}$ such that $\left|g_{1}(x)\right| \leq 1$, the bound $\int_{1}^{x}\left|g_{1}(u)\right| \log u \mathrm{~d} \Pi(u) \ll x$ holds, and $\int_{1}^{\infty} x^{-1}\left|g_{2}(x)\right| \mathrm{d} \Pi(x)<\infty$. Then, for real constants $c, \alpha$, and a slowly varying function $L(u)$ with $|L(u)|=1$, the relation

$$
\int_{1}^{x} \frac{G(u)}{u} \mathrm{~d} u=\frac{c x^{1+i \alpha}}{(1+i \alpha)^{2}} L(\log x)+o(x)
$$

is satisfied if and only if

$$
\widehat{G}(s)=\frac{c}{s-1-i \alpha} L\left(\frac{1}{\sigma-1}\right)+o\left(\frac{1}{\sigma-1}\right)
$$

holds uniformly for $t$ in compact intervals.

The asymptotic relation (2.2) could be differentiated via elementary familiar arguments (e.g. [10, Section I.18, p. 37]) if $G$ satisfies additional Tauberian hypotheses. For example, if $g(u) \geq 0$, so that $G$ is non-decreasing, we must essentially have $\alpha=0$ and $L(u)=1$ in (2.2); one then deduces $G(x) \sim c x$. We might apply this to $G(x)=N(x)$ itself; the next corollary clarifies even more why the hypotheses on $N$ in Theorem 1.1 are redundant.

Corollary 2.2. Assume that the Chebyshev upper bound (1.1) holds. Then, for $a>0$, $N(x) \sim$ ax holds if and only if

$$
\int_{1}^{\infty} \frac{|N(x)-a x|}{x^{\sigma+1}} \mathrm{~d} x=o\left(\frac{1}{\sigma-1}\right) .
$$

These relations are also equivalent to

$$
\zeta(s)=\frac{a}{s-1}+o\left(\frac{1}{\sigma-1}\right),
$$

uniformly for $t$ on compact intervals.

Proof. The implications $N(x) \sim a x \Rightarrow(2.4) \Rightarrow(2.5)$ trivially hold unconditionally. Assume now that (2.5) holds, then in particular $\zeta(\sigma) \sim a /(\sigma-1)$ and the HardyLittlewood-Karamata theorem yields logarithmic density,

$$
\int_{1^{-}}^{x} \frac{\mathrm{d} N(u)}{u} \sim a \log x
$$

By Theorem 2.1, we have $\int_{1}^{x} N(u) / u \mathrm{~d} u \sim a x$. As explained above, using that $N$ is non-decreasing one concludes that $N(x) \sim a x$, as required.

Furthermore, 
Theorem 2.3. Assume $N$ has positive density (1.2). Let $\mathrm{d} G=\exp ^{*}(g \mathrm{~d} \Pi)$ be a multiplicative arithmetic measure with $g=g_{1}+g_{2}$ such that $\left|g_{1}(x)\right| \leq 1$, the bound $\int_{1}^{x}\left|g_{1}(u)\right| \log u \mathrm{~d} \Pi(u) \ll x$ holds, and $\int_{1}^{\infty} x^{-1}\left|g_{2}(x)\right| \mathrm{d} \Pi(x)<\infty$. Then, for real constants $c, \alpha$, and a slowly varying function $L(u)$ with $|L(u)|=1$, the asymptotic relation

$$
G(x)=\frac{c x^{1+i \alpha}}{1+i \alpha} L(\log x)+o(x)
$$

is satisfied if and only if (2.3) holds uniformly for $t$ in compact intervals.

Proof. The non-trivial implication is (2.3) implies (2.7). Set $\mathrm{d} G_{i}=\exp ^{*}\left(g_{i} \mathrm{~d} \Pi\right)$ and in addition consider the convolution inverse of $\mathrm{d} G_{2}$, that is, $\mathrm{d} F=\exp ^{*}\left(-g_{2} \mathrm{~d} \Pi\right)$. Since $\widehat{F}(s)$ is absolutely convergent on $\Re e s=1$, a small computation shows that $(\widehat{F}(s)-\widehat{F}(1+i \alpha)) /(s-1-i \alpha)=o(1 /(\sigma-1))$ uniformly for $t$ on compacts. Thus, with the same uniformity, (2.3) yields

$$
\widehat{G_{1}}(s)=\widehat{G}(s) \widehat{F}(s)=\frac{c_{1}}{s-1-i \alpha} L\left(\frac{1}{\sigma-1}\right)+o\left(\frac{1}{\sigma-1}\right),
$$

where $c_{1}=c / \widehat{G}_{2}(1+i \alpha)$. Applying Theorem 2.1 to $\mathrm{d} G_{1}$, we obtain

$$
\int_{1}^{x} \frac{G_{1}(u)}{u} \mathrm{~d} u=\frac{c_{1} x^{1+i \alpha}}{(1+i \alpha)^{2}} L(\log x)+o(x) .
$$

Let us verify that $G_{1}(x) / x$ is slowly oscillating (in the sense of Schmidt, cf. [10, Def. I.16.1, p. 32]). Due to our hypothesis on $G_{1}$ it is clear that $\left|\mathrm{d} G_{1}\right| \leq \mathrm{d} N$. Hence, if $\eta>1$,

$$
\left|\frac{G_{1}(\eta x)}{\eta x}-\frac{G_{1}(x)}{x}\right| \leq(\eta-1)\left|\frac{G_{1}(\eta x)}{\eta x}\right|+\frac{N(\eta x)-N(x)}{x} \ll \eta-1+o_{\eta}(1),
$$

by (1.2). Since $L(\log x)$ is slowly varying, a standard elementary Tauberian argument gives

$$
G_{1}(x)=\frac{c_{1} x^{1+i \alpha}}{1+i \alpha} L(\log x)+o(x) .
$$

The asymptotic formula (2.2) then follows from a variant of Wintner's mean-value theorem (i.e., Lemma 3.4(i) below).

Combining Theorem 2.3 with [5, Lemma 3.6], we immediately obtain Corollary 1.2 with $\mathrm{d} M$ the convolution inverse of $\mathrm{d} N$, namely, the measure $\mathrm{d} M=\exp ^{*}(-\mathrm{d} \Pi)$ (for discrete number systems $M$ is then the sum function of the Beurling analog of the Möbius function). It is worth pointing out that the hypothesis $N(x) \sim a x$ cannot be omitted in Corollary [1.2, as shown by [5, Examples 4.2 and 4.3]. We also mention that one can construct examples of number systems for which $M(x)=o(x)$ and (1.2) hold for some $a>0$, but for which the Chebyshev bound (1.1) fails; see for instance Kahane's example [6, Example 3.6].

The ensuing version of the Halász mean-value theorem holds true.

Theorem 2.4. Assume the positive density condition (1.2) and the Chebyshev upper bound (1.1) and let $\mathrm{d} G=\exp ^{*}(g \mathrm{~d} \Pi)$ be a multiplicative arithmetic measure such that $g=g_{1}+g_{2}$ with $\left|g_{1}(x)\right| \leq 1$ and $\int_{1}^{\infty} x^{-1}\left|g_{2}(x)\right| \mathrm{d} \Pi(x)<\infty$. 
If there is $\alpha \in \mathbb{R}$ such that

$$
\int_{1}^{\infty} \frac{1-\Re e\left(g(x) x^{-i \alpha}\right)}{x} \mathrm{~d} \Pi(x)
$$

converges, then

$$
G(x)=\frac{x^{1+i \alpha}}{1+i \alpha} \exp \left(-\int_{1}^{x} \frac{1-g(u) u^{-i \alpha}}{u} \mathrm{~d} \Pi(u)\right)+o(x) .
$$

Otherwise, if there is no such $\alpha$, then $G$ has zero mean-value,

$$
G(x)=o(x) .
$$

In either case, there are real constants $c, \alpha$, and a slowly varying function $L(u)$ with $|L(u)|=1$ such that (2.7) holds.

Proof. Using again Lemma 3.4(i), we may assume that $g_{2}=0$. The result can then be deduced from Theorem 2.3 along the same lines of the proof of the corresponding Halász mean-value theorem for the natural numbers given e.g. in Elliott's book [8, Chapter 6]. Therefore, we only give a brief sketch and leave most details to the reader. When (2.8) diverges for every $\alpha$, the classical argument involving Dini's theorem yields $\widehat{G}(s)=o(1 /(\sigma-1))$ (see e.g. [17, Lemma 3.1], one just uses here $\zeta(\sigma) \ll 1 /(\sigma-1)$ ), so that we obtain (2.10) via Theorem 2.3 with $c=0$.

In the case of convergence of (2.8), one may assume $\alpha=0$, because a simple integration by parts computation then yields the general result. We note that an adapted version of [8, Lemma 6.8, p. 242] holds in view of $\psi(x) \ll x$, while [8, Lemma 6.9, p. 243] is valid because of $N(x) \sim a x$ in the form (2.5). Hence, similarly as in [8, pp. 245-246], one derives that (2.3) holds for $t$ on compacts with $\alpha=0$ and a slowly varying function $L$ with modulus 1 satisfying

$$
c L(\log x)=\exp \left(\int_{1}^{\infty} \frac{g(u)-1}{u} \exp \left(-\frac{\log u}{\log x}\right) \mathrm{d} \Pi(u)\right)+o(1) .
$$

By Theorem 2.3, it thus just remains to verify that the latter integral expression equals

$$
\int_{1}^{x} \frac{g(u)-1}{u} \mathrm{~d} \Pi(u)+o(1) .
$$

But this can also be established reasoning as in [8, pp. 246-247] with the aid of $\psi(x) \ll$ $x$ and the simple bound

$$
\int_{1}^{x} \frac{\log u}{u} \mathrm{~d} \Pi(u)=\frac{\psi(x)}{x}+\int_{1}^{x} \frac{\psi(u)}{u^{2}} \mathrm{~d} u \ll \log x .
$$

As a simple corollary, one also obtains Wirsing's mean-value theorem in this context. Of course, Corollary 1.2 is also a consequence of it. 
Corollary 2.5. Suppose the positive density condition (1.2) and the Chebyshev upper bound (1.1) hold. Let $\mathrm{d} G=\exp ^{*}(g \mathrm{d \Pi})$ be a real-valued multiplicative arithmetic measure such that $g=g_{1}+g_{2}$ with $\left|g_{1}(x)\right| \leq 1$ and $\int_{1}^{\infty} x^{-1}\left|g_{2}(x)\right| \mathrm{d} \Pi(x)<\infty$. Then,

$$
\lim _{x \rightarrow \infty} \frac{G(x)}{x}=\exp \left(-\int_{1}^{\infty} \frac{1-g(x)}{x} \mathrm{~d} \Pi(x)\right),
$$

where the right-hand side is taken as zero when the integral diverges.

Proof. Indeed, the convergent case directly follows from Theorem 2.4. Assume thus that (2.8) diverges for $\alpha=0$. If it also diverges for all other values of $\alpha$, we are done as well since (2.10) holds. If (2.8) converges for some $\alpha \neq 0$, then (2.7) holds for some $c \in \mathbb{R}$ and $L$. We need to show that necessarily $c=0$. If $c$ were not zero, we would have

$$
\lim _{x \rightarrow \infty} \frac{G\left(x e^{\frac{\pi}{2 \alpha}}\right)}{G(x)}=i e^{\frac{\pi}{2 \alpha}}
$$

but this limit must be real so that one must either have $c=0$ or that such an $\alpha$ does not exist.

We end this section with a remark concerning the case of discrete generalized number systems.

Remark 2.6. All the results from this section cover the particular instance of multiplicative functions on a discrete generalized number system satisfying $\left|f\left(n_{k}\right)\right| \leq 1$ for every generalized integer $n_{k}$, provided the generalized number system has a positive density and a Chebyshev upper bound holds for the generalized primes.

Given a multiplicative function $f$, the associated multiplicative measure is $\mathrm{d} G=$ $f \mathrm{~d} N$. The functions $f$ and $g$ in the representation $f \mathrm{~d} N=\exp ^{*}(g \mathrm{~d} \Pi)$ determine one another by their values on generalized prime powers linked by means of the relations

$$
1+\sum_{\nu=1}^{\infty} \frac{f\left(p_{k}^{\nu}\right)}{p_{k}^{s \nu}}=\prod_{\nu=1}^{\infty} \exp \left(\frac{g\left(p_{k}^{\nu}\right)}{\nu p_{k}^{\nu s}}\right),
$$

which are obtained by comparing factors corresponding to each generalized prime $p_{k}$ in $\sum_{k=0}^{\infty} n_{k}^{-s} f\left(n_{k}\right)=\exp \left(\int_{1}^{\infty} x^{-s} g(x) \mathrm{d} \Pi(x)\right)$ with its Euler product. Taylor expanding the exponential and multiplying out the right-hand side of (2.11), one readily deduces that

$$
f\left(p_{k}^{\nu}\right)=\sum_{\nu=1 \cdot m_{1}+2 \cdot m_{2}+\cdots+\nu \cdot m_{\nu}} \prod_{j=1}^{\nu} \frac{1}{m_{j} !}\left(\frac{g\left(p_{k}^{j}\right)}{j}\right)^{m_{j}} .
$$

In particular, $g\left(p_{k}\right)=f\left(p_{k}\right)$ for every generalized prime. The formula (2.12) can be rewritten in terms of the (exponential) complete Bell polynomials (see e.g. [3, p. 134], where the notation $Y_{n}=B_{n}$ is employed),

$$
f\left(p_{k}^{\nu}\right)=\frac{1}{\nu !} B_{\nu}\left(0 ! g\left(p_{k}\right), 1 ! g\left(p_{k}^{2}\right), \ldots,(\nu-1) ! g\left(p_{k}^{\nu}\right)\right) .
$$


Conversely, taking logarithms in (2.11) and using [3, Theorem A, p. 140],

$$
g\left(p_{k}^{\nu}\right)=\sum_{j=1}^{\nu}(-1)^{j-1} \frac{(j-1) !}{(\nu-1) !} B_{\nu, j}\left(1 ! f\left(p_{k}\right), 2 ! f\left(p_{k}^{2}\right), \ldots,(\nu-j+1) ! f\left(p_{k}^{\nu-j+1}\right)\right),
$$

where the $B_{\nu, j}$ stand for the partial Bell polynomials. In particular, if $f$ is completely multiplicative, we have $g\left(p_{k}^{\nu}\right)=f\left(p_{k}^{\nu}\right)$ for each $k$ and $\nu \geq 1$. In view of $\left|f\left(n_{k}\right)\right| \leq 1$, we find using (2.13) and [3, Eq. (3h), Theorem B, p. 135]

$$
\left|g\left(p_{k}^{\nu}\right)\right| \leq \sum_{j=1}^{\nu} \frac{(j-1) !}{(\nu-1) !} B_{\nu, j}(1 !, 2 !, \ldots,(\nu-j+1) !)=2^{\nu}-1 .
$$

We further decompose $g=\tilde{g}+h$ with

$$
\tilde{g}\left(p_{k}^{\nu}\right)= \begin{cases}g\left(p_{k}^{\nu}\right) & \text { if } p_{k}>2 \\ 0 & \text { otherwise }\end{cases}
$$

It is clear that the multiplicative arithmetic measure $\mathrm{d} \tilde{G}=\exp ^{*}(\tilde{g} \mathrm{~d} \Pi)$ satisfies the hypotheses we have been considering in this section. The Mellin transform of $\mathrm{d} H=$ $\exp ^{*}(h \mathrm{~d} \Pi)$ is simply the Euler product

$$
\widehat{H}(s)=\prod_{p_{k} \leq 2}\left(1+\sum_{\nu=1}^{\infty} \frac{f\left(p_{k}^{\nu}\right)}{p_{k}^{\nu s}}\right)
$$

which is obviously absolutely convergent for $\sigma>0$. Using that $\widehat{H}(s) \ll 1$ and $\widehat{H}^{\prime}(s) \ll 1$ on the half-plane $\sigma \geq 1$, the proof of Theorem 2.1 we give in Section 4 can readily be adapted to obtain (2.2) for $G(x)=\sum_{n_{k} \leq x} f\left(n_{k}\right)$ from (2.3) $),\left|f\left(n_{k}\right)\right| \leq 1$, positive density $N(x) \sim a x$, and the Chebyshev upper bound. On the other hand, the conditions $\left|f\left(n_{k}\right)\right| \leq 1$ and $N(x) \sim a x$ imply that $G(x) / x$ is slowly oscillating, so that Theorem 2.3 is valid in this case. Applying Theorem 2.4 to $\tilde{G}$ and then Lemma 3.4)(i) to $\mathrm{d} G=\mathrm{d} H * \mathrm{~d} \tilde{G}$, the Halász mean-value theorem takes the form: If there is $\alpha \in \mathbb{R}$ such that

$$
\sum_{k=1}^{\infty} \frac{1-\Re e\left(p_{k}^{-i \alpha} f\left(p_{k}\right)\right)}{p_{k}}
$$

converges, then

$$
\frac{1}{x} \sum_{n_{k} \leq x} f\left(n_{k}\right)=\frac{x^{i \alpha}}{1+i \alpha} \prod_{p_{k} \leq x}\left(1-\frac{1}{p_{k}}\right)\left(1+\sum_{\nu=1}^{\infty} \frac{f\left(p_{k}^{\nu}\right)}{p_{k}^{\nu(1+i \alpha)}}\right)+o(1) ;
$$

otherwise, $f$ has zero mean-value. Moreover, the assertion in Corollary 2.5 becomes: If in addition $f$ is real-valued, we always have

$$
\lim _{x \rightarrow \infty} \frac{1}{x} \sum_{n_{k} \leq x} f\left(n_{k}\right)=\prod_{k=1}^{\infty}\left(1-\frac{1}{p_{k}}\right)\left(1+\sum_{\nu=1}^{\infty} \frac{f\left(p_{k}^{\nu}\right)}{p_{k}^{\nu}}\right) .
$$




\section{Auxiliary elementary estimates}

We start with a key estimate based on Rankin's method (cf. [14, Section II.3]).

Proposition 3.1. Let $\mathrm{d} G=\exp ^{*}(g \mathrm{~d} \Pi)$ be a multiplicative arithmetic measure such that

$$
\int_{1}^{x}|g(u)| \log u \mathrm{~d} \Pi(u) \ll x \log ^{\beta} x
$$

with $\beta \geq 0$. Then,

$$
\frac{G(x)}{x} \ll \log ^{\beta-1} x \exp \left(\int_{1}^{x} \frac{|g(u)|}{u} \mathrm{~d} \Pi(u)\right) .
$$

Proof. We first estimate $\int_{1}^{x} \log u|\mathrm{~d} G(u)|$. Note that the multiplication by log operator is a derivation on the convolution algebra of measures [7, Section 2.8]. We have $\log \cdot \mathrm{d} G=$ $\mathrm{d} G *(g \cdot \log \mathrm{d} \Pi)$ and so (3.1) yields

$$
\int_{1}^{x} \log u|\mathrm{~d} G(u)| \ll \int_{1^{-}}^{x} \frac{x}{u} \log ^{\beta}\left(\frac{x}{u}\right)|\mathrm{d} G(u)| \ll x \log ^{\beta} x \exp \left(\int_{1}^{x} \frac{|g(u)|}{u} \mathrm{~d} \Pi(u)\right),
$$

where we have used that multiplying by $1 / u$ commutes with the exponential of measures. We now apply Rankin's trick,

$$
\begin{aligned}
G(x) & \ll \int_{1}^{\sqrt{x}} \log u|\mathrm{~d} G(u)|+\frac{2}{\log x} \int_{\sqrt{x}}^{x} \log u|\mathrm{~d} G(u)| \\
& \ll\left(\sqrt{x} \log ^{\beta} x+x \log ^{\beta-1} x\right) \exp \left(\int_{1}^{x} \frac{|g(u)|}{u} \mathrm{~d} \Pi(u)\right) .
\end{aligned}
$$

From here we deduce:

Corollary 3.2. Suppose that $\int_{1}^{x} u^{-1} \mathrm{~d} \Pi(u) \leq \log \log x+O(1)$. If $\mathrm{d} G=\exp ^{*}(g \mathrm{~d} \Pi)$ is such that

$$
\int_{1}^{x}|g(u)| \log u \mathrm{~d} \Pi(u) \ll x
$$

then

$$
\int_{\Re e s=\sigma}\left|\frac{\widehat{G}(s)}{s}\right|^{2}|\mathrm{~d} s| \ll \int_{0}^{\infty} e^{-2 y(\sigma-1)} \exp \left(2 \int_{1}^{e^{y}} \frac{|g(u)|-1}{u} \mathrm{~d} \Pi(u)\right) \mathrm{d} y
$$

as $\sigma \rightarrow 1^{+}$, and

$$
\int_{\Re e s=\sigma}\left|\frac{\widehat{G}^{\prime}(s)}{s \widehat{G}(s)}\right|^{2}|\mathrm{~d} s| \ll \frac{1}{\sigma-1} .
$$


Proof. Proposition 3.1 gives (3.4). The bound (3.5) then follows from the Plancherel identity because for fixed $\sigma$ the function $\widehat{G}(s) / s$ is the Fourier transform of $G\left(e^{y}\right) e^{-\sigma y}$. Next, $-\widehat{G}^{\prime}(s) / \widehat{G}(s)$ is the Mellin transform of the measure $g(u) \log u \mathrm{~d} \Pi(u)$, whose primitive is $O(x)$ by (3.3). So, the Plancherel theorem implies (3.6).

Let us point out that $\int_{1}^{x} u^{-1} \mathrm{~d} \Pi(u) \leq \log \log x+O(1)$ implies upper logarithmic density $\int_{1}^{x} u^{-1} \mathrm{~d} N(u) \ll \log x$. Moreover, the condition (2.1) turns out to be equivalent to a weak form of Mertens' formula.

Lemma 3.3. (2.1) holds if and only if

$$
\int_{1}^{x} \frac{\mathrm{d} \Pi(u)}{u}=\log \log x+O(1) .
$$

In addition, these relations are equivalent to $\zeta(\sigma) \asymp 1 /(\sigma-1)$.

Proof. Combine [7, Proposition 4.5 and Theorem 4.7] for (2.1) if and only if (3.7). The equivalence between (2.1) and $\zeta(\sigma) \asymp 1 /(\sigma-1)$ follows from [7, Proposition 4.2, Proposition 4.8, and Corollary 4.10].

We refer the reader to [7, 13] for more information on Mertens type results for Beurling numbers.

We shall also need the ensuing simple lemma. Note that part (i) is a version of Wintner's mean-value theorem.

Lemma 3.4. Let $A$ and $B$ be two functions of local bounded variation on $[1, \infty)$ such that $\int_{1-}^{\infty} u^{-1}|\mathrm{~d} A(u)|<\infty$. Consider $\mathrm{d} D=\mathrm{d} A * \mathrm{~d} B$. Given a slowly varying function $\ell$ with $|\ell(u)|=1$ and $\alpha, b \in \mathbb{R}$, we have:

(i) $B(x)=b x^{1+i \alpha} \ell(x)+o(x)$ implies $D(x)=\widehat{A}(1+i \alpha) b x^{1+i \alpha} \ell(x)+o(x)$.

(ii) $\int_{1}^{x} u^{-1} B(u) \mathrm{d} u=b x^{1+i \alpha} \ell(x)+o(x)$ implies

$$
\int_{1}^{x} \frac{D(u)}{u} \mathrm{~d} u=\widehat{A}(1+i \alpha) b x^{1+i \alpha} \ell(x)+o(x) .
$$

Proof. For (i), we have

$$
\frac{1}{x^{1+i \alpha} \ell(x)} \int_{1^{-}}^{x} \mathrm{~d} A * \mathrm{~d} B=\int_{1^{-}}^{x} \frac{B(x / u)}{(x / u)^{1+i \alpha} \ell(x / u)} \frac{\ell(x / u)}{\ell(x)} \frac{\mathrm{d} A(u)}{u^{1+i \alpha}} \rightarrow b \int_{1^{-}}^{\infty} \frac{\mathrm{d} A(u)}{u^{1+i \alpha}} .
$$

For (ii), we notice that

$$
\int_{1}^{x} \frac{B(u)}{u} \mathrm{~d} u=\int_{1^{-}}^{x} \log (x / u) \mathrm{d} B(u)=\int_{1^{-}}^{x} \mathrm{~d} H * \mathrm{~d} B,
$$

with $\mathrm{d} H(u)=u^{-1} \chi_{[1, \infty)}(u) \mathrm{d} u$. So,

$$
\int_{1}^{x} \frac{D(u)}{u} \mathrm{~d} u=\int_{1^{-}}^{x} \mathrm{~d} H * \mathrm{~d} B * \mathrm{~d} A,
$$

whence we conclude that part (ii) is a special case of part (i).

Finally, we translate (2.2) into another weighted average for $\mathrm{d} G$. 
Lemma 3.5. Let $G$ be a function of local bounded variation on $[1, \infty)$ such that $\int_{1}^{x} u^{-1} G(u) \mathrm{d} u=o(x \log x)$. Consider

$$
F(x)=\int_{1}^{x}\left(\int_{1}^{u} \log y \mathrm{~d} G(y)\right) \frac{\mathrm{d} u}{u} .
$$

Then, for $\ell$ slowly varying with $|\ell(u)|=1$ and some constant $c \in \mathbb{R}$,

$$
\int_{1}^{x} \frac{G(u)}{u} \mathrm{~d} u=\operatorname{cx\ell }(x)+o(x) \quad \text { if and only if } \quad F(x)=c x \ell(x) \log x+o(x \log x) .
$$

Proof. Integrating by parts, $\int_{1}^{x} u^{-1} G(u) \mathrm{d} u \sim \operatorname{cx} \ell(x)$ is equivalent to

$$
\int_{1}^{x} \log u \frac{G(u)}{u} \mathrm{~d} u \sim \operatorname{cx\ell }(x) \log x .
$$

We also have

$$
F(x)=\int_{1}^{x} \log u \frac{G(u)}{u} \mathrm{~d} u-\int_{1}^{x} \frac{1}{u} \int_{1}^{u} \frac{G(y)}{y} \mathrm{~d} y \mathrm{~d} u=\int_{1}^{x} \log u \frac{G(u)}{u} \mathrm{~d} u+o(x \log x),
$$

whence the claim follows.

\section{Proof of Theorem 2.1}

We start with some reductions. We only need to show that (2.3) implies (2.2). The same reasoning employed at the beginning of the proof of Theorem 2.3 and Lemma 3.4(ii) allow us to assume without loss of generality that $g_{2}=0$. So, our hypotheses on $g$ are $|g(x)| \leq 1$ and (3.3). We may also assume that $\alpha=0$, namely, we are supposing that

$$
\widehat{G}(s)=\frac{c}{s-1} L\left(\frac{1}{\sigma-1}\right)+o\left(\frac{1}{\sigma-1}\right),
$$

uniformly for $t$ on compact intervals. In view of Lemma 3.3, the bound (3.4) from Corollary 3.2 applies, so $G(x) \ll x$. Therefore, $G$ fulfills the conditions of Lemma 3.5 and from now on we can restrict our attention to the function $F$ defined in (3.8).

We should prove that

$$
F(x)=c x L(\log x) \log x+o(x \log x) .
$$

The Mellin-Stieltjes transform of the function $F$ is $\widehat{F}(s)=-\widehat{G}^{\prime}(s) / s$. Given $x>e$, it is convenient to set $\sigma_{x}=1+1 / \log x$. By the Perron inversion formula, we have

$$
\frac{F(x)}{x}=-\frac{1}{2 \pi i} \int_{\Re e s=\sigma_{x}} \frac{x^{s-1} \widehat{G}^{\prime}(s)}{s^{2}} \mathrm{~d} s .
$$

Next, we take a large number $\lambda>1$, fixed for the while. We split the integral over the line $\left\{s: \Re e s=\sigma_{x}\right\}$ into three pieces, taken over

$$
\begin{aligned}
& \Gamma_{0}=\left\{\sigma_{x}+i t:|t| \leq \lambda / \log x\right\}, \\
& \Gamma_{1}=\left\{\sigma_{x}+i t: \lambda / \log x<|t| \leq \lambda\right\}, \\
& \Gamma_{2}=\left\{\sigma_{x}+i t: \lambda<|t|\right\} .
\end{aligned}
$$


The integral over $\Gamma_{0}$ can easily be handled using the condition (4.1) and the fact that $L$ is slowly varying; proceeding exactly as in [8, p. 239], we obtain

$$
-\frac{1}{2 \pi i} \int_{\Gamma_{0}} \frac{x^{s-1} \widehat{G}^{\prime}(s)}{s^{2}} \mathrm{~d} s=c L(\log x) \log x+O\left(\frac{\log x}{\lambda}\right) .
$$

We now employ (3.6) and the Cauchy-Schwarz inequality in order to get

$$
\int_{\Gamma_{j}} \frac{x^{s-1} \widehat{G}^{\prime}(s)}{s^{2}} \mathrm{~d} s \ll \log ^{1 / 2} x\left(\int_{\Gamma_{j}}\left|\frac{\widehat{G}(s)}{s}\right|^{2}|\mathrm{~d} s|\right)^{1 / 2}, \quad j=1,2 .
$$

It remains to estimate the latter two integrals. For the integral over the unbounded intervals $\Gamma_{2}$, we can apply (3.5) to the multiplicative arithmetic measures

$$
\exp ^{*}\left(u^{ \pm i(\lambda+m)} g(u) \mathrm{d} \Pi(u)\right) \text {; }
$$

hence,

$$
\begin{aligned}
& \int_{\Gamma_{2}}\left|\frac{\widehat{G}(s)}{s}\right|^{2}|\mathrm{~d} s| \leq \sum_{m=0}^{\infty} \frac{1}{1+(\lambda+m)^{2}}\left(\int_{-\lambda-m-1}^{-\lambda-m}+\int_{\lambda+m}^{\lambda+m+1}\right)\left|\widehat{G}\left(\sigma_{x}+i t\right)\right|^{2} \mathrm{~d} t \\
& \leq 5 \sum_{m=0}^{\infty} \frac{1}{1+(\lambda+m)^{2}} \int_{\Re e s=\sigma_{x}}\left(\left|\frac{\widehat{G}(s+i(\lambda+m))}{s}\right|^{2}+\left|\frac{\widehat{G}(s-i(1+\lambda+m))}{s}\right|^{2}\right)|\mathrm{d} s| \\
& \ll \frac{1}{\lambda} \int_{0}^{\infty} e^{-2\left(\sigma_{x}-1\right) y} \mathrm{~d} y=O\left(\frac{\log x}{\lambda}\right) .
\end{aligned}
$$

On the other hand, using (4.1),

$$
\int_{\Gamma_{1}}\left|\frac{\widehat{G}(s)}{s}\right|^{2}|\mathrm{~d} s| \leq\left(|c| \frac{\log x}{\lambda}+o_{\lambda}(\log x)\right)^{1 / 2} \int_{\Re e s=\sigma_{x}}\left|\frac{(\widehat{G}(s))^{3 / 4}}{s}\right|^{2}|\mathrm{~d} s| .
$$

To deal with the last integral we notice that $(\widehat{G}(s))^{3 / 4}$ is the Mellin transform of the arithmetic measure $\exp ^{*}(3 g / 4 \mathrm{~d} \Pi)$. Applying Plancherel's identity and (3.2) to this measure, using the upper bound from (3.7) and the hypothesis $|g(u)| \leq 1$, we obtain

$$
\begin{aligned}
\int_{\Re e s=\sigma_{x}}\left|\frac{(\widehat{G}(s))^{3 / 4}}{s}\right|^{2}|\mathrm{~d} s| & \ll \int_{0}^{\infty} e^{-2 y\left(\sigma_{x}-1\right)} y^{-2} \exp \left(2 \int_{1}^{e^{y}} \frac{(3 / 4)|g(u)|}{u} \mathrm{~d} \Pi(u)\right) \mathrm{d} y \\
& \ll \int_{0}^{\infty} e^{-2 y\left(\sigma_{x}-1\right)} y^{-1 / 2} \mathrm{~d} y \ll \log ^{1 / 2} x .
\end{aligned}
$$

Collecting all estimates, we arrive at

$$
\frac{F(x)}{x \log x L(\log x)}-c \ll \frac{1}{\lambda^{1 / 4}}+o_{\lambda}(1) .
$$

Taking first the limit superior as $x \rightarrow \infty$ and then $\lambda \rightarrow \infty$, we have shown (4.2). This establishes Theorem 2.1. 
Remark 4.1. It is worth pointing out that we have not used the lower bound from (3.7) in this section. Thus, our proof above shows that Theorem 2.1 still holds true if the hypothesis (2.1) is relaxed to $\int_{1}^{x} u^{-1} \mathrm{~d} \Pi(u) \leq \log \log x+O(1)$.

\section{REFERENCES}

[1] P. T. Bateman, H. G. Diamond, Asymptotic distribution of Beurling's generalized prime numbers, in: Studies in number theory, W. J. LeVeque, ed., pp. 152-210, Mathematical Association of America, 1969.

[2] A. Beurling, Analyse de la loi asymptotique de la distribution des nombres premiers généralisés, Acta Math. 68 (1937), 255-291.

[3] L. Comtet, Advanced combinatorics. The art of finite and infinite expansions, D. Reidel Publishing Co., Dordrecht, 1974.

[4] H. Daboussi, K. H. Indlekofer, Two elementary proofs of Halász's theorem, Math. Z. 209 (1992), $43-52$.

[5] G. Debruyne, H. G. Diamond, J. Vindas, $M(x)=o(x)$ Estimates for Beurling numbers, J. Théor. Nombres Bordeaux 30 (2018), 469-483.

[6] G. Debruyne, J. Vindas, On Diamond's $L^{1}$ criterion for asymptotic density of Beurling generalized integers, Michigan Math. J. 68 (2019), 211-223.

[7] H. G. Diamond, W.-B. Zhang, Beurling generalized numbers, Mathematical Surveys and Monographs series, American Mathematical Society, Providence, RI, 2016.

[8] P. D. T. A. Elliott, Probabilistic number theory. I. Mean-value theorems. Grundlehren der Mathematischen Wissenschaften, 239, Springer-Verlag, New York-Berlin, 1979.

[9] G. Halász, Über die Mittelwerte multiplikativer zahlentheoretischer Funktionen, Acta Math. Acad. Sci. Hungar. 19 (1968), 365-403.

[10] J. Korevaar, Tauberian theory. A century of developments, Grundlehren der Mathematischen Wissenschaften, Vol. 329, Springer-Verlag, Berlin, 2004.

[11] L. Lucht, K. Reifenrath, Mean-value theorems in arithmetic semigroups, Acta Math. Hungar. 93 (2001), 27-57.

[12] H. L. Montgomery, R. C. Vaughan, Multiplicative number theory. I. Classical theory, Cambridge Studies in Advanced Mathematics, 97, University Press, Cambridge, 2007.

[13] P. Pollack, On Mertens' theorem for Beurling primes, Canad. Math. Bull. 56 (2013), 829-843.

[14] W. Schwarz, J. Spilker, Arithmetical functions. An introduction to elementary and analytic properties of arithmetic functions and to some of their almost-periodic properties, London Mathematical Society Lecture Note Series, 184, Cambridge University Press, Cambridge, 1994.

[15] G. Tenenbaum, Introduction to analytic and probabilistic number theory, Graduate Studies in Mathematics, 163, American Mathematical Society, Providence, RI, 2015.

[16] W.-B. Zhang, A generalization of Halász's theorem to Beurling's generalized integers and its application, Illinois J. Math. 31 (1987), 645-664.

[17] W.-B. Zhang, Halász's theorem for Beurling numbers, Acta Arith. 183 (2018), 223-235.

Department of Mathematics: Analysis, Logic and Discrete Mathematics, Ghent University, KriJgslaan 281, B 9000 Ghent, Belgium

E-mail address: gregory.debruyne@UGent. be

E-mail address: frederick.maes@UGent.be

E-mail address: jasson.vindas@UGent.be 